\title{
Acción directiva: Un enfoque centrado en competencias
}

\author{
Zambrano Flores, Blanca M. * \\ Marval Galvis, Elina V. **
}

\begin{abstract}
Resumen
La acción directiva se debate entre el mecanicismo que la ha caracterizado y la exigencia de una administración alternativa. Se trata de desarrollar competencias articuladas a los saberes, vistas como una intención que constituye una "disposición para" lograr cambios y desempeños exitosos. El objetivo de la investigación es proponer un enfoque centrado en competencias para viabilizar la acción directiva en los Programas Académicos del pregrado en la Universidad Nacional Experimental "Rafael María Baralt". El estudio es descriptivo, orientado como un proyecto factible. La técnica de recolección de datos fue la encuesta. La población estuvo conformada por 3 Directores de Programas a los cuales se les aplicó el instrumento denominado ECAD. Los resultados destacan: la realidad dialógica entre la cual se debate el director por asumir el cambio o mantener su acción en la cotidianidad administrativa de la universidad. En conclusión, se requiere una acción directiva centrada en el humanismo que incluya una visión amplia de la nueva realidad administrativa, desarrollada con apropiación del contexto nacional, regional, local e institucional para favorecer el uso pleno de las competencias.
\end{abstract}

Palabras clave: Administración, programas académicos, acción directiva, competencias, saberes.

* Dra. en Educación, mención Administración Educativa. Profesora Titular de la UNERMB. Ex Vicerrectora Académica. Profesora meritoria CONABA Nivel I.

E-mail: blancazambranodec@cantv.net.

** Dra. en Ciencias de la Educación. Profesora Titular de la UNERMB. Investigadora acreditada por el Programa de Promoción al Investigador PPI. Profesora meritoria CONABA Nivel I.

E-mail: elinavictoria@cantv.net. 


\title{
Directive Action: An Approach Centered on Competencies
}

\begin{abstract}
Directive action is debated between the mechanicalism that has characterized it and the requirement for an alternative humanist administration. It is about developing competencies linked to knowledge, viewed as an intention that constitutes "willingness to" bring about changes and successful performances. The aim of this research is to propose an approach focused on competencies, in order to make directive action viable in undergraduate academic programs at the Experimental National University "Rafael María Baralt." The study is descriptive, targeted as a feasible project. The datacollecting technique was the survey. The population was composed of three directors of programs to which the tool called CBA was applied. Results highlight: the dialogic reality through which the director debates about assuming the change or maintaining his action in the everyday university administration. In conclusion, a directive action focused on humanism is required that includes a broad vision of the new administrative reality, developed with appropriation of the national, regional, local and institutional environment to encourage the full use of competencies.
\end{abstract}

ey ords: Administration, academic programs, directive action, competencies, types of knowledge.

\section{Introducción}

La universidad constituye un fenómeno social integrado a la sociedad. Hoy, dado los cambios producidos en el mundo, recibe cada vez mayores exigencias, no sólo asociadas al desarrollo económico, sino también a elementos de la vida política y cultural de un país, por ello es importante considerar, la globalización, su multiplicidad de manifestaciones, la asunción de nuevos paradigmas en la ciencia y en los distintos ámbitos del quehacer humano, lo cual crea diversas exigencias, como la formación del talento humano y la búsqueda de desempeños superiores en el ámbito organizacional.

Al tratarse de la universidad y su compromiso para generar los cambios y transformaciones requeridas en el mundo, esta búsqueda resulta además de ineludible, perentoria. La acción directiva como proceso de carácter social humanista y creativo, constituye una opción para alcanzar los desempeños esperados. Razón que justifica el conocimiento de la administración y su instrumental, entre las cuales se destacan las funciones del proceso administrativo: planificar, organizar, dirigir y controlar, no de forma mecanicista, sino con una concepción amplia, humanista e integradora.

Lo anterior, alude al estudio de las competencias, asociadas a los cuatro (4) pilares sugeridos por la United Nations for Education Science and Culture Organizations (UNESCO) para ser incorporados a la educación, esto es: saber conocer, saber hacer, saber ser y saber convivir. Saberes pertinentes en el desempeño de actividades en cualquier área del conocimiento; la administración universitaria no constituye la excepción.

En ese contexto las exigencias provenientes del entorno repercuten en su interior, acrecentando requerimientos para abordar el conocimiento con estrategias que garanticen el aprendizaje, pero a 
Acción directiva: Un enfoque centrado en competencias

Zambrano Flores, Blanca M. y Marval Galvis, Elina V.

su vez demandan procesos administrativos cada vez más eficientes en los cuales son fundamentales las competencias para lograr desempeños acordes con las necesidades planteadas.

Resulta común en el contexto organizacional, la expresión "el problema es gerencial". Es decir, la multiplicidad de problemas cuya explicación se asocia a la administración y/o gerencia se endosan a su actor principal, indistintamente de la denominación recibida: presidente, director, gerente, coordinador, y a la forma como éste ejecuta las funciones del proceso administrativo.

La universidad como parte de las instituciones sociales tiene responsabilidades con respecto a la sociedad que se expresan, entre otros elementos, en servir de punto de encuentro con el resto del mundo, coadyuva así a interpretarla, consolidarla y transformarla.

Lo anterior implica superar esquemas tradicionales, no solo relativas a sus funciones básicas: docencia, investigación y extensión o a cambios radicales para la producción y/o aprehensión del conocimiento, sino a la adopción de procesos administrativos tendentes a desarrollarlos en el marco de suficiencia en las competencias del talento humano, implica pues, el ejercicio de una acción directiva plena, integral que viabilice conjugar en la práctica pensamiento y acción.

Integrar ambos elementos, para la transformación de procesos inherentes a lo administrativo en el ámbito universitario exige, formación en competencias, vistas no de forma acrítica o instrumental tras la búsqueda de la eficacia o eficiencia administrativa. Esto ratifica la importancia de las competencias en el talento humano para lograr el desarrollo de procesos administrativos alejados de la racionalidad tradicional que mediatiza la acción directiva y la circunscribe a un conjunto de técnicas y procedimientos en buena proporción desprovista de la visión humanista, integral, holística, necesarias para direccionar, dada la complejidad del mundo moderno.

La universidad tiene responsabilidad más allá de su mejoramiento interno; por cuanto al ser productora del saber, no debe escatimar esfuerzos para direccionar la construcción colectiva, la responsabilidad social y ética, pero fundamentalmente la formación de talento humano, con competencias y sentido ético para abordar su crecimiento, el establecimiento de relaciones dialógicas tanto a lo interno como a lo externo y la generación de respuestas a situaciones concretas.

La búsqueda a la cual se hace referencia implica a lo interno un nuevo rumbo en las funciones universitarias: docencia, investigación y extensión, cuya direccionalidad se debe gestar desde su interior, es decir, de forma endógena. El talento humano constituye a la luz de sus resultados como instancia productora del saber y de la investigación una de sus fortalezas. Sin embargo, pareciera contradictorio, pues la crítica de distintos sectores a su gestión administrativa no se corresponden con esa apreciación, muy por el contrario se le acusa de "burocrática, funcional y mecánica" alejada de prácticas tendentes a dirigir el desarrollo de competencias en las personas mediante el trabajo coordinado.

La universidad venezolana ha sido objeto de señalamientos de todo orden para cuestionar su funcionamiento, entre 
ellos los relativos a su administración, se le endosa burocracia, lentitud e ineficiencia en los procesos administrativos; si bien esa realidad no es del todo cierta, de su dinámica se desprenden elementos que reflejan la necesidad de cambios.

En consecuencia, las competencias del talento humano vistas como disposición inherente a la condición humana cuyo desarrollo facilita un desempeño idóneo, constituyen característica sine qua non en la administración de las universidades. En la Universidad Nacional Experimental "Rafael María Baralt" (UNERMB) esa realidad no es distinta, es referencia para la ejecución de este estudio, cuyo objetivo es proponer un enfoque centrado en competencias para viabilizar la acción directiva de quienes tienen esa responsabilidad en los Programas Académicos, en los cuales se imparte formación profesional en pregrado en la UNERMB.

El estudio es descriptivo con la modalidad de proyecto factible, en la intención de generar una propuesta para viabilizar la acción directiva centrada en competencias para ser aplicada en los Programas Académicos: Administración, Educación e Ingeniería de la UNERMB. Se asume una perspectiva holística, transdisciplinaria, compleja en la intención de proponer un enfoque centrado en competencias humanísticas y cognitivas necesarias en la acción directiva.

Abordar la investigación desde la perspectiva descriptiva, mediante la modalidad de proyecto factible, se justifica básicamente por dos (2) razones: en primer lugar, por cuanto ninguna ciencia tiene métodos absolutamente propios o exclusivos, de manera que el método científico proviene de la combinación de un pequeño número de procedimientos de investigación. En segundo lugar, la modalidad de proyectos factibles, constituye una valiosa alternativa para aportar soluciones a la problemática que se infiere.

La población estuvo conformada por la totalidad de quienes direccionan los Programas Académicos de la Universidad Nacional Experimental Rafael María Baralt, donde se imparte formación profesional en el área de pregrado, constituida por tres (3) directores los cuales representan la unidad de información, por ello el estudio es censal. Es importante destacar que la UNERMB, creada por decreto presidencial No. 1435 el 15 de marzo de 1982, en su proyecto de creación, contempla sólo tres Programas de pregrado: Administración, Educación e Ingeniería, contexto donde se produce la acción directiva, objeto de este estudio.

La técnica de recolección fue la encuesta y el instrumento un cuestionario estructurado, denominado Enfoque de competencias en la acción directiva (ECAD), elaborado con la experiencia y conocimiento previo de las investigadoras en torno a las variables en estudio, así como el nivel de información y comprensión de los entrevistados con respecto al tema. El cuestionario agrupó una serie de interrogantes relativas a las competencias y la acción directiva, cuyos elementos conceptuales fueron cuidadosamente relacionados con: las fases del proceso administrativo (planificación, organización, dirección, control) y los saberes: saber conocer, saber hacer, saber ser y saber convivir. 
Acción directiva: Un enfoque centrado en competencias

Zambrano Flores, Blanca M. y Marval Galvis, Elina V.

\section{Bases teóricas}

Las teorías que se presentan a continuación sustentan las variables objeto de estudio.

\subsection{Dirección y acción directiva}

La dirección es el elemento del proceso administrativo responsable de coordinar el talento humano en las empresas. Es fundamental, pues su racionalidad viabiliza alcanzar lo planificado, dada la autoridad y la coordinación de actividades que permite. Las habilidades humanas presentes en la dirección, comunicación, liderazgo, motivación, toma de decisiones y, en el nuevo contexto, la integración, la ratifican como el corazón del proceso administrativo.

El carácter dinámico de dicha función o proceso, permite asumir el modelo humanista, en contraposición al mecanicista cuyos postulados aun se intentan superar y con el cual se identifican en mayor proporción las restantes etapas del proceso administrativo. La dirección reviste un carácter eminentemente humano, exige para la solución de problemas integrar el tiempo y las circunstancias, es necesario interactuar día a día, minuto a minuto, no sólo a partir de políticas, planes, programas y procedimientos, sino además debe regirse por valores, premisas y principios que en todo caso guían la acción directiva en una perspectiva humanista. Al respecto, Ballina (2000:91) explica "toda dirección está influenciada por la época en la cual opera".

Se asume la acción directiva como aquella encaminada a "lograr de forma creativa, sistémica y humanista la aplica- ción del proceso administrativo: planificar, organizar, dirigir y controlar, en la intención de lograr una combinación armónica de los recursos técnicos, económicos, financieros y el talento humano de los cuales dispone una organización, lo cual puede ser posible mediante el desarrollo de competencias" (Zambrano, 2003: 39). Esto coincide con las ideas de Chiavenato (2002:494) quien afirma "los más recientes enfoques administrativos destacan que las personas hacen las diferencias en las organizaciones".

Las competencias son importantes para ejercer la acción directiva en instituciones de educación superior, pues como sostiene Pérez (2005:19) "el directivo junto con su planta académica y administrativa, debe establecer e implantar una visión única, práctica, compartida, acorde con los lineamientos oficiales y de la comunidad". Percepción que ratifica el papel del entorno en la administración interna de la organización o institución de la cual se trate.

Lo planteado conduce a estudiar las competencias, en cualquier ámbito de desempeño del ser humano, el educativo y en particular, la educación superior no es la excepción, tal como refiere Bunk (citado en Maldonado, 2006:32) "la educación superior tiene gran responsabilidad en cuanto al desarrollo de la personalidad, el cambio del comportamiento y el desarrollo de la eficiencia", agrega el autor su hipótesis en torno a que este nivel educativo desarrolla "la competencia profesional, la metódica, lo social y la cooperativa". Si bien en el campo empresarial se presentan los enfoques por competencias como una opción para aumentar su rentabilidad, competitividad y por ende su 
supervivencia, también la función pública busca elevar la capacidad institucional a través de la mejora en los procedimientos administrativos.

Eso hace evidente la búsqueda de enfoques más allá de lo normativo y el creciente interés en la formación de las competencias de los servidores públicos, tal como refleja la variedad de talleres, cursos u otras opciones destinadas a lograr ese objetivo. Una visión particular, en el caso de este nivel académico es lograr sinergia entre la formación profesional y el crecimiento personal; ello posiblemente siente las bases para desarrollar competencias más allá de lo cognitivo expresado en una práctica concreta, no es posible obviar, el señalamiento de Wolf (citado en Maldonado, 2006:40) "la competencia es una abstracción y no algo que podemos observar directamente", se localiza en lo profundo de la personalidad, se materializa en el comportamiento y se exhibe mediante el desempeño.

Es oportuno reflexionar en torno al personal directivo en las instituciones de educación superior; sector no sólo direccionado por talento humano, sino sus actores fundamentales: docentes-estudiantes y la multiplicidad de interacciones establecidas al interior y a lo externo de la universidad, presentan el mismo carácter, como refiere Guédez (1998:268) "ciertamente los recursos tecnológicos, financieros y materiales son importantes, pero sólo tienen sentido y funcionan a partir del equipo humano de una organización y particularmente del directivo", por cuanto también en la universidad en los últimos años es del manejo colectivo la tesis de que la misma requiere de gerentes.
En la universidad tradicionalmente se hablaba de administradores, hoy como consecuencia del proceso modernizador, estos actores se denominan gerentes, tal como expresa Villarroel (2005) "quieren ser gerentes y reciben cursos al respecto; se les paga como gerentes, pero siguen siendo unos simples administradores, en el concepto tradicional". La crítica no es gratuita deviene de la burocracia y la ineficiencia administrativa que a decir de diversos estudiosos presenta la administración y/o gerencia universitaria.

Para el autor la dinámica universitaria no contribuye con el proceso de cambio, su administrador tiene como propósito conservar la caracterización "esencial" de la institución no necesita proyectos de transformación universitaria; por el contrario la misión y visión institucional es sustituida por la fragmentada circunstancia de la cotidianidad.

La importancia de la universidad en la sociedad del conocimiento para el desarrollo sostenible y sustentable del país hacen obvia la necesidad de competencias para permitir los cambios. El gerente no está como señala Villarroel (2005) para cuidar la universidad, no en el sentido tradicional del administrador, sino para transformarla. Lo citado permite afirmar que las competencias resultan para el desempeño la mejor opción, porque son contrarias a la fragmentación y viabilizan la armonía necesaria entre el saber conocer, saber hacer, saber ser y saber convivir, pilares fundamentales para la UNESCO de perfiles por competencias.

La acción directiva ejercida por quien funcionalmente realice esa actividad en la empresa, implica tomar conciencia del cambio operado en los distin- 
Acción directiva: Un enfoque centrado en competencias Zambrano Flores, Blanca M. y Marval Galvis, Elina V.

tos países del mundo, ello es la respuesta a las necesidades humanas, de lo cual se infiere que no sólo atiende a esas necesidades, también la acción. Pero ésta última engloba al tratarse del ser, pensamientos y sentimientos cuya manifestación se da a través de sus competencias (Diagrama1).

\subsection{Competencias: de sus orígenes al concepto}

El concepto de competencias lo planteó por primera vez en la lingüística Noam Chomsky en 1965, para explicar cómo es posible comunicarse mediante la apropiación del lenguaje. Por su parte, Gerhard Bunk, en los años 70 introduce el término en el mundo educativo y laboral; clasificándolas en competencias técnicas, metodológicas, sociales y participativas, lo cual se asume en el estudio por su vinculación con la formación, el trabajo y la aplicación de los saberes.

A nivel empresarial su uso se generaliza, de forma implícita se aportan elementos que sugieren la relación entre la formación y el mundo del trabajo así como, la sinergia a lograr entre el conocer, el saber, el hacer y el ser.

Para Núñez, las competencias (2000:22) constituyen un "conjunto de conocimientos, habilidades, destrezas y actitudes cuya aplicación en el trabajo se traduce en un desempeño superior, que contribuye al logro de los objetivos clave del negocio". Definición que refleja cuatro (4) elementos: (a) conocimiento, saber conocer; (b) habilidades y destrezas, saber hacer; (c) actitudes, saber ser y (d) saber convivir. Estos elementos armonizan en quien ejecuta la acción, conocimiento y sentimiento, necesarios en el desempeño (Diagrama 2).

Se asume la concepción de competencias de Bunk (citado en Tobón, 2006:25) quien las define "en relación con los sujetos que la poseen o disponen de los conocimientos, destrezas y actitudes, para el ejercicio de una profesión, así como para resolver los problemas internos y externos a la organización". Se complementa al integrar los saberes: (a) saber conocer; (b) saber hacer, (c) saber ser y (d) saber convivir (Diagrama 3).

\section{Diagrama 1 \\ Interacción de saberes y desempeño}

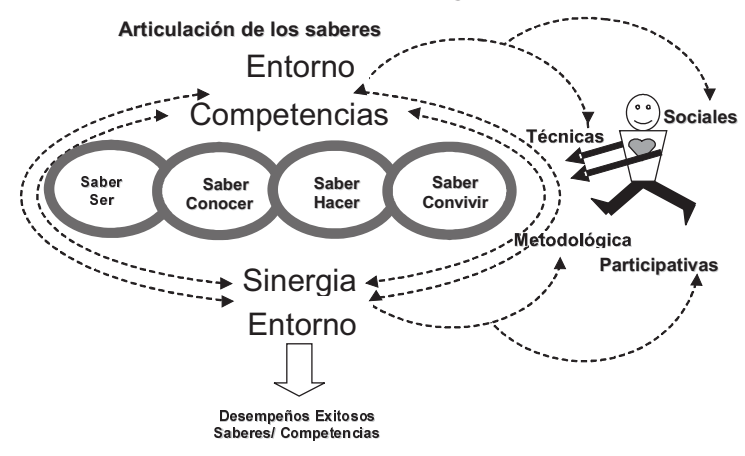

Fuente: Elaboración propia. 
Diagrama 2

Definición de competencias

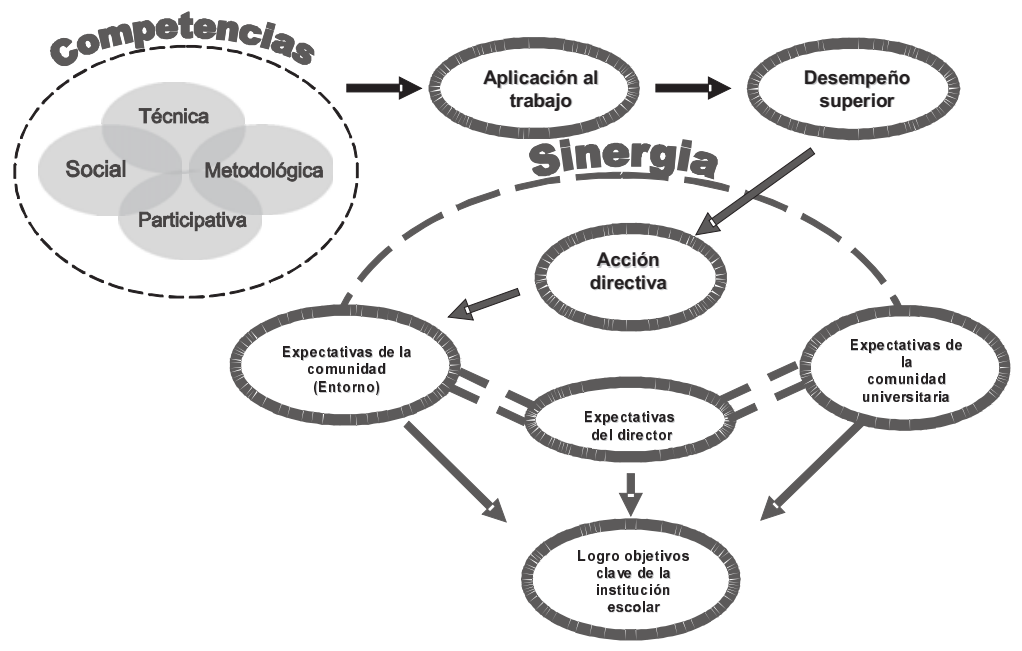

Fuente: Elaboración propia.

Diagrama 3

Competencias de sus elementos

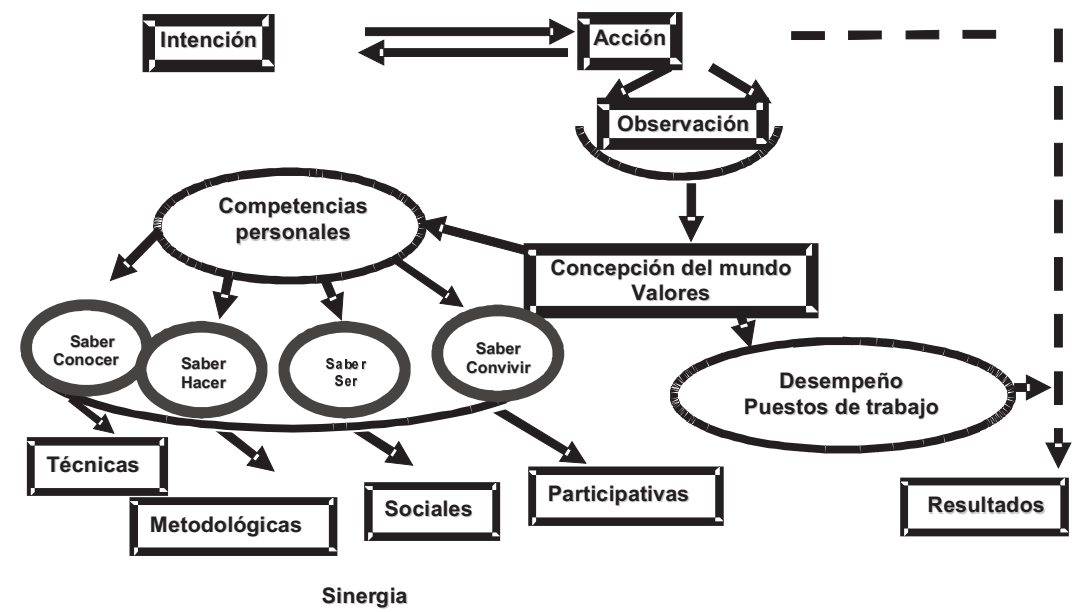

Fuente: Elaboración propia. 
Acción directiva: Un enfoque centrado en competencias

Zambrano Flores, Blanca M. y Marval Galvis, Elina V.

Dirigir por competencias plantea cambios y desempeños exitosos, "depende de su concepción y de su manifestación en la función directiva" (Zambrano, 2006b:21). Para Benavides (2002) su interpretación y aplicación depende del autor con el cual se identifique, de las tendencias culturales, contextos de origen, aplicación y la racionalidad organizacional en la cual se soporta.

Esa dimensión de las competencias crea nuevos espacios, posibilidades y escenarios para la acción directiva, como plantea Zambrano (2006b:16) "en el marco de una visión holística, sistémica, sostenible, compleja, participativa e integrativa, norte de los procesos de cambios en el mundo". De allí que Bunk (citado en Tobón, 2006) establece la relación entre la pedagogía y el trabajo, para resumir el significado de las competencias, articular ambos componentes y analizar si el trabajo opera como objeto, medio o forma de educación.

Se entiende por pedagogía del trabajo, "modalidad de aprender a trabajar que toma en consideración todos los aspectos del ser humano" (Bunk, citado en Tobón, 2006:26); se asume el trabajo como una actividad consciente que armoniza lo cognitivo, la motricidad y la afectividad. Los objetivos, cambios de comportamiento y desarrollo de la eficiencia (cualificación), se expresan en el aprendizaje mediante cambios en el comportamiento, evidentes en la autodeterminación, responsabilidad y cogestión. En el desarrollo de la eficiencia, objetivo básico para la formación del trabajador es imprescindible la cualificación, flexibilidad, humanismo y participación. Se infiere de los objetivos en la propuesta de Bunk, la taxonomía de competencias técnicas, metodológicas, sociales y participativas, las cuales se definen a continuación:

1. Competencias técnicas: reflejan dominio, experticia o destreza de las tareas y contenidos de su ámbito de trabajo. Significa poseer conocimientos y relacionarlos con un determinado ámbito profesional que permitan dominar como experto los contenidos y tareas acordes a su actividad laboral.

2. Competencias metodológicas: resultan de saber reaccionar al aplicar las tareas que corresponden a una actividad determinada, o a las irregularidades o desviaciones presentes. Significa aplicar los conocimientos a situaciones concretas, utilizar procedimientos adecuados, solucionar problemas y transferir con ingenio las experiencias adquiridas a situaciones novedosas.

3. Competencias sociales: la posee quien colabora con otras personas de forma comunicativa, su comportamiento orienta al grupo y genera entendimiento interpersonal. Significa estar atento a la evolución del mercado laboral.

4. Competencias participativas: saber participar, decidir y asumir responsabilidades en un puesto de trabajo. Significa tomar decisiones y relativizar posibles frustraciones (Cuadro 1).

Las competencias técnicas, metodológicas, sociales y participativas, se asumen tal como se explica a lo largo del estudio, asociadas a los cuatro (4) pilares propuestos en el Informe Delors, presentado por la UNESCO (1996) los cuales a juicio de las investigadoras se asocian a 


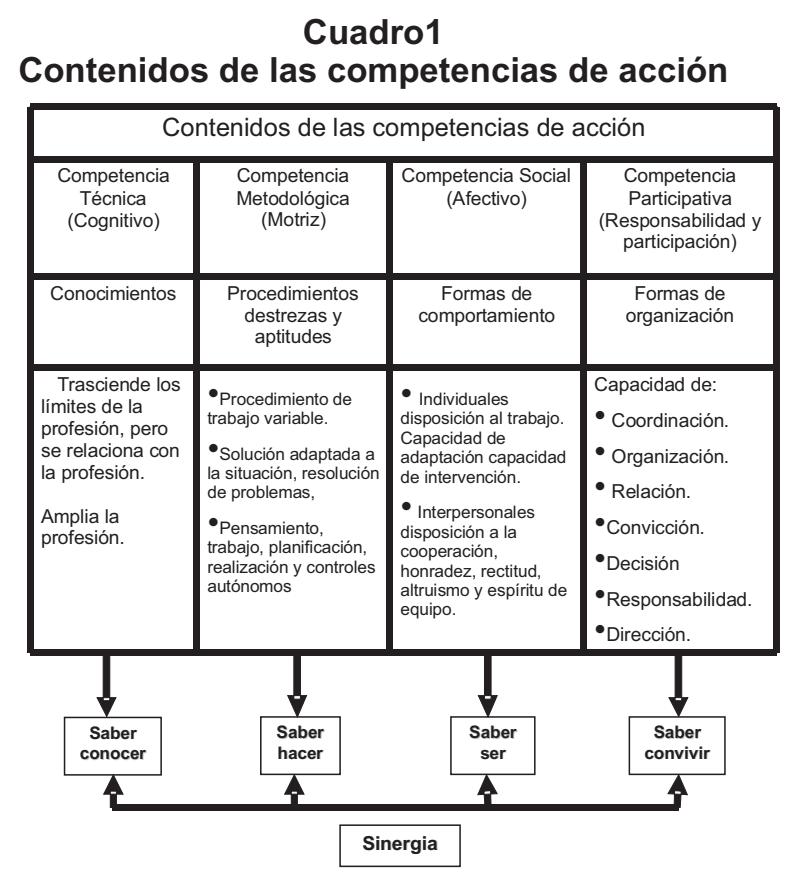

Fuente: Elaboración propia con base en Maldonado (2006). Las competencias, una opción de vida

los objetivos pedagógicos de carácter cognitivo, motriz, afectivo, de responsabilidad y compromiso ético contenidos en la propuesta de Bunk.

\subsection{Competencias y saberes: una relación indiscutible}

A continuación se definen los saberes necesarios para formar los ciudadanos y profesionales del siglo que recién se inicia, estos son:

Aprender a conocer. supone la capacidad de aprendizaje para adquirir conocimientos requeridos en su profesión, así como la apropiación de los instrumentos necesarios para comprender el mundo, vivir con dignidad, desarrollar sus competencias profesionales $\mathrm{y}$, comunicarse con los demás. En este caso, se adquieren habilidades del pensamiento relativas a la comprensión, el análisis, la síntesis, la abstracción y la generalización.

Aprender a hacer: resulta inseparable del aprender a conocer, esto es obvio por cuanto nadie hace algo sobre lo cual no conoce. Implica la práctica, el aplicar principios, leyes y experiencias en tareas o exigencias concretas del vivir o del mundo del trabajo. En cierta forma equivale a las acciones necesarias en el desempeño de ciertas competencias para el ejercicio de las profesiones.

Aprender a convivir: supone la formación para la cooperación y aprender a vivir juntos, se atiende el concepto de identidad personal, aprender a ubicarse en el lugar del otro y atenuar el potencial 
Acción directiva: Un enfoque centrado en competencias

Zambrano Flores, Blanca M. y Marval Galvis, Elina V.

de autodestrucción acumulado por la humanidad. Implica prepararse para enfrentar y resolver conflictos, contrarrestar la competitividad mediante acciones de solidaridad, participar en proyectos comunitarios que faciliten el acercamiento entre las personas, acentuar la comprensión por el otro y la defensa de los derechos ajenos como si fuesen suyos. Prepara para trabajar por objetivos comunes con visión ecológica y desarrollar convicciones tendentes a lograr sostenibilidad y sustentabilidad.

Se resalta la importancia de todos los saberes, pero este último es significativo por cuanto la educación superior ha centrado sus principios en una visión individualista y fragmentaria, no solo de las acciones sino de los contenidos. Lograr el cambio cultural que implica la transición de una sociedad mecanicista a una sociedad humanista con promoción de la equidad y de la inclusión, donde prive el interés colectivo sobre el individual, exige fortalecer el aprender a convivir.

Aprendera ser: se asocia con el desarrollo de las capacidades cognitivas, psicomotoras, afectivas, sociales, sensibilidad, responsabilidad, sentido estético, de pertenencia a un terruño, una nación, al planeta; incentiva a querer como consecuencia cuidar y conservar para las generaciones futuras.

En ese contexto, se observa la articulación a la cual se hace referencia en el estudio pero, además se considera que cada uno de estos saberes se compone de procesos, los cuales constituyen operaciones donde la esencia es la estructura y el procesamiento de la información, esto resulta común y automático en los seres humanos aunque su desarrollo se dé en distinto grado, dado su asociación a las potencialidades y oportunidades ofrecidas por el entorno. Esta perspectiva implica considerar que para lograr un desempeño exitoso es necesario en el marco de los enfoques por competencia, la articulación de los saberes.

\section{Análisis de los resultados}

En el instrumento ECAD, la parte denominada Competencias centradas en los saberes, presenta unos enunciados para cada función del proceso administrativo, asociados a los saberes de quien ejecuta la acción directiva con relación a su desempeño. Las respuestas de las alternativas consideradas o no por el director en cada una de las funciones se agruparon de acuerdo a su identificación con los saberes (conocer, hacer, ser y convivir), esto permitió establecer el tipo de competencia (técnica, metodológica, social y participativa) que prevalece en la acción directiva de la cual trata el estudio.

En cuanto a las competencias desarrolladas en el proceso de planificación, el $66.66 \%$ de los directores considera que su acción interpreta las necesidades del entorno, mientras el $100 \%$ afirma estudiar planes operativos, todas éstas relacionadas con el saber conocer. Los resultados evidencian fortaleza en cuanto a este saber, pues al menos dos (2) de ellos consideraron realizar estas actividades al planificar, es decir el $66.66 \%$. Es importante destacar que de las 10 alternativas presentadas, dos (2) legitiman el saber conocer, de las cuales sólo la relativa a estudiar planes operacionales la ejecutan todos, ello es reflejo de la presencia de competencias técnicas. 
Con relación al saber hacer, el 33.33\% expresó manejar una distribución más justa de los recursos, diseñar planes para lograr cambio de actitud, utilizar el tiempo como variable importante en su gestión y diseñar los objetivos del programa bajo su responsabilidad, mientras el $66.66 \%$ afirmó desarrollar estrategias para asumir el cambio. Los resultados constituyen una debilidad pues prevalece el $33.33 \%$ en casi todas las respuestas, esto significa que cada competencia en torno a los enunciados relacionados con este saber, sólo es considerado por uno de los directores.

De lo planteado se deriva la competencia metodológica en una baja proporción, a pesar de su importancia en la acción directiva, lo cual permite suponer que ésta es guiada por la intuición y no por una clara comprensión de la disponibilidad de los saberes como estrategias para lograr en el marco de las competencias, desempeños exitosos.

En cuanto al saber ser, el $33.33 \%$ manifestó asumir el personal operativo como fuente de creatividad y democratizar los planes. Los resultados constituyen una debilidad ya que cada competencia en torno a sus enunciados, es considerado sólo por uno de los directores. Se reflejan en consecuencia, competencias sociales cuyo reforzamiento es necesario, sobre todo al tomar en cuenta que para la planificación se presentan (2) alternativas y aún así cada una es ejecutada sólo por uno de ellos.

Con relación al saber convivir, el $66.66 \%$ participa de las responsabilidades atribuidas a los niveles bajo su conducción, razón por la cual constituye una fortaleza pues la mayoría asume esta competencia asociada a las participativas e indiscutiblemente también requieren ser reforzadas, fundamentalmente por la importancia del convivir en el ámbito organizacional y social.

En consecuencia, predomina en la función planificación, el saber conocer asociado a competencias técnicas relacionadas con procesos como análisis, comprensión, síntesis, abstracción y generalización, propias en este caso de las ciencias administrativas y en particular, de la planificación. Esto se ratifica por cuanto de las 10 alternativas, ejecutan en un $100 \%$ la referida a estudiar planes operacionales; posiblemente el no considerar las otras opciones también importantes está asociado al desconocimiento en la materia, pues tal como expresaron sus conocimientos administrativos fueron adquiridos a través de la autoformación, quizás insuficiente para un desempeño exitoso (ver Tabla 1).

En cuanto a las competencias desarrolladas en la organización, el 66.66\% afirmó estudiar la organización del trabajo y consideran que su acción directiva integra asuntos de la cotidianidad administrativa mediante planes y objetivos. Por su parte, el $100 \%$ expresó conocer la estructura organizativa. Estas alternativas están relacionadas con el saber conocer. Los resultados obtenidos constituyen una fortaleza media, pues se puede observar que dos (2) de los directores consideraron realizar estas actividades, ello implica competencias técnicas.

Con relación al saber hacer, el $33.33 \%$ expresó que diseña los ambientes de trabajo, mientras el $66.66 \%$ facilita la integración organizacional, desarrolla métodos de trabajo y aplica la jerarquía 
Acción directiva: Un enfoque centrado en competencias

Zambrano Flores, Blanca M. y Marval Galvis, Elina V.

Tabla 1

Competencias a desarrollar en el proceso de planificación

\begin{tabular}{|c|c|c|c|c|c|c|}
\hline \multirow[b]{2}{*}{ Competencias } & \multicolumn{2}{|c|}{ Considera } & \multicolumn{3}{|c|}{ No considera } & \multirow[b]{2}{*}{ Total $(\%)$} \\
\hline & $\mathbf{F}$ & $\%$ & Saberes & $\mathbf{F}$ & $\%$ & \\
\hline $\begin{array}{l}\text { Interpreta las necesidades } \\
\text { del entorno }\end{array}$ & 2 & 66.66 & Conocer & 1 & 33.33 & 100 \\
\hline $\begin{array}{l}\text { Maneja distribución más } \\
\text { justa de los recursos }\end{array}$ & 1 & 33.33 & Hacer & 2 & 66.66 & 100 \\
\hline $\begin{array}{l}\text { Desarrolla estrategias } \\
\text { para asumir el cambio }\end{array}$ & 2 & 66.66 & Hacer & 1 & 33.33 & 100 \\
\hline $\begin{array}{l}\text { Asume personal como } \\
\text { fuente de creatividad }\end{array}$ & 1 & 33.33 & Ser & 2 & 66.66 & 100 \\
\hline $\begin{array}{l}\text { Estudia planes } \\
\text { Operacionales }\end{array}$ & 3 & 100 & Conocer & - & - & - \\
\hline $\begin{array}{l}\text { Participa de las } \\
\text { responsabilidades de } \\
\text { niveles bajo su conducción }\end{array}$ & 2 & 66.66 & Convivir & 1 & 33.33 & 100 \\
\hline Democratiza los planes & 1 & 33.33 & Ser & 2 & 66.66 & 100 \\
\hline $\begin{array}{l}\text { Diseña planes para cambiar } \\
\text { Actitudes }\end{array}$ & 1 & 33.33 & Hacer & 2 & 66.66 & 100 \\
\hline $\begin{array}{l}\text { Utiliza tiempo como variable } \\
\text { Importante }\end{array}$ & 1 & 33.33 & Hacer & 2 & 66.66 & 100 \\
\hline Diseña objetivos del Programa & 1 & 33.33 & Hacer & 2 & 66.66 & 100 \\
\hline Todas las anteriores & - & - & - & - & - & - \\
\hline Ningunas de las anteriores & - & - & - & - & - & - \\
\hline
\end{tabular}

Fuente: Elaboración Propia basada en los datos obtenidos del instrumento ECAD.

administrativa. Los resultados con este saber se pueden considerar una fortaleza, pues al no estar en la ejecución de las tareas, las restantes sugerencias en sí no constituyen lo antes expresado, esto significa comprensión en torno a los enunciados de este saber, por dos (2) de los directores, lo cual se traduce en competencias sociales también necesarias de reforzar.

En cuanto al saber ser, el $66.66 \%$ fomenta el trabajo en equipo, mientras el $100 \%$ expresó que generan sinergia en las diferentes instancias administrativas. Los resultados relacionados con este saber constituyen una fortaleza por cuanto cada enunciado es considerado por al menos dos (2) de los directores. Sin embargo, es obvia la necesidad de fortalecer las mismas, sobre todo de asociarlas de formas directamente proporcional con los saberes.

Para el saber convivir, los resultados demuestran que el $66.66 \%$ participa en equipos de trabajo, razón por la cual constituye una fortaleza, pues al menos 
dos (2) del total de la población realiza esta actividad. Se destaca la disposición a compartir en equipo como un elemento conveniente para promover el trabajo cooperativo y participativo, en consecuencia esto sienta las bases para la promoción de una cultura organizacional integrada y no fragmentaria.

En cuanto al proceso de organización, de las 10 alternativas planteadas en términos de las competencias a desarrollar por los directores en su acción. Los mismos centran su atención en la ejecución de las tareas propias del proceso, pues conocen la estructura organizativa de la institución y estimulan la sinergia entre las instancias administrativas. De esto se desprende en términos generales, limitaciones en torno a esta función, por cuanto se concentran en el plano meramente administrativo y las dos (2) alternativas enunciadas, demostrándose en la práctica de la cotidianidad administrativa contradicciones en cuanto a la sinergia que dicen generar. El análisis previo demuestra la ejecución de tareas en las cuales se traducen competencias técnicas y sociales, éstas últimas con la contradicción antes expuesta (Tabla 2).

En cuanto a los resultados del proceso administrativo representado por la dirección y las competencias para realizar esta función, se tiene que el $66.66 \%$ interpretan debidamente la delegación de autoridad y conocen las necesidades institucionales. Estas alternativas en el marco de las competencias están relacionadas con el saber conocer. Los resultados

Tabla 2

Competencias a desarrollar en el proceso de organización

\begin{tabular}{lcccccc}
\hline & \multicolumn{7}{c}{ Considera } & \multicolumn{5}{c}{ No considera } \\
\hline \multicolumn{1}{c}{ Competencias } & F & $\%$ & Saberes & F & $\%$ & Total (\%) \\
\hline Facilita la integración & 2 & 66.66 & Hacer & 1 & 33.33 & 100 \\
Fomenta trabajo en equipo & 2 & 66.66 & Ser & 1 & 33.33 & 100 \\
Integra asuntos de la & & & & & & \\
cotidianidad administrativa & 2 & 66.66 & Conocer & 1 & 33.33 & 100 \\
Estudia organiz. del trabajo & 2 & 66.66 & Conocer & 1 & 33.33 & 100 \\
Desarrolla métodos de trabajo & 2 & 66.66 & Hacer & 1 & 33.33 & 100 \\
Conoce la estructura Organizativa & 3 & 100 & Conocer & - & - & - \\
Participa en equipos de Trabajo & 2 & 66.66 & Convivir & 1 & 33.33 & 100 \\
Aplica la jerarquía administ. & 2 & 66.66 & Hacer & 1 & 33.33 & 100 \\
Genera sinergia & 3 & 100 & Ser & - & - & - \\
Diseña ambientes & 1 & 33.33 & Hacer & 2 & 66.66 & 100 \\
Todas las anteriores & - & - & - & - & - & - \\
Ninguna de las anteriores & - & - & - & - & - & - \\
\hline
\end{tabular}

Fuente: Elaboración Propia basada en los datos obtenidos del instrumento ECAD. 
Acción directiva: Un enfoque centrado en competencias Zambrano Flores, Blanca M. y Marval Galvis, Elina V.

constituyen una fortaleza, pues ya que dos (2) de los directores realizan estas actividades.

En tal sentido, es importante reflexionar en torno al logro, pues ello representa el conocimiento expresado en términos de las competencias y se observa en el desempeño. Las competencias en este caso se asocian a las técnicas, vistas desde el punto de vista holístico.

Con relación al saber hacer, el $33.33 \%$ facilita la comunicación con el entorno, mientras el $100 \%$ expresó que facilitan la producción académica de las personas bajo su responsabilidad y diseñan políticas para promover la integración entre la gente. Los resultados revelan en la primera de las opciones una debilidad, pues sólo uno de ellos señaló realizarla, en la segunda constituye una fortaleza al mostrar la ejecución de la tarea por parte de los tres (3) directores.

En cuanto al saber ser, el $33.33 \%$ fomenta una buena comunicación a lo interno, el $66.66 \%$ demuestra sensibilidad ante las necesidades de las personas indistintamente de su posición en la organización y asume críticas a su gestión, mientras el $100 \%$ afronta con serenidad las dificultades institucionales. Los resultados con relación al proceso de comunicación interna muestran una debilidad que genera preocupación por cuanto ésta se asocia en el estudio, a la transferencia de significados y de valores. En consecuencia, asumir esto refleja dificultades institucionales al respecto y la necesidad de promover en la actuación del director formas de comportamientos favorables a la adquisición y consolidación en su práctica adminis- trativa de las competencias sociales y el enriquecimiento del ser.

En relación con el saber convivir, no hubo respuestas, esto indica que ninguno participa en la orientación hacia la tarea y las relaciones, sugerencia presentada en torno al convivir directamente relacionada con las competencias participativas. Los resultados muestran debilidad dentro de la organización, pues deja a un lado el convivir como elemento fundamental para promover la sinergia, condición necesaria en quien direcciona en el ámbito organizacional. La individualidad que caracteriza la acción administrativa en cada uno de los programas objeto de estudio da cuenta de ello.

Los resultados en cuanto a la dirección como función eminentemente humana propia del proceso administrativo Ilaman la atención, por cuanto en este caso el saber hacer, expresado en las competencias metodológicas prevalece con respecto a los otros saberes. Se infiere entonces el "cómo", el cual no es más que la construcción del hombre, por cuanto efectivamente la ciencia administrativa aporta en este caso "el qué" y "el para qué", de allí la influencia del pensamiento, carga valorativa o en todo caso la concepción del mundo de quienes ejecutan la acción directiva. Es importante además generar acciones tendentes a armonizar los saberes y lograr en quienes dirigen comprensión en torno a la disponibilidad de los mismos para viabilizar su uso en función de las competencias (Tabla 3).

En torno al proceso de control, las respuestas arrojan que el $66.66 \%$ estudia aspectos relativos al desempeño del per- 
Tabla 3

Competencias a desarrollar en el proceso de dirección

\begin{tabular}{|c|c|c|c|c|c|c|}
\hline \multirow[b]{2}{*}{ Competencias } & \multicolumn{2}{|c|}{ Considera } & \multicolumn{3}{|c|}{ No considera } & \multirow[b]{2}{*}{ Total $(\%)$} \\
\hline & $\mathbf{F}$ & $\%$ & Saber & $\mathbf{F}$ & $\%$ & \\
\hline Facilita la producción académica & 3 & 100 & Conocer & - & - & 100 \\
\hline Conoce las necesidades institucionales & 2 & 66.66 & Conocer & 1 & 33.33 & 100 \\
\hline Demuestra sensibilidad & 2 & 66.66 & Ser & 1 & 33.33 & 100 \\
\hline $\begin{array}{l}\text { Afronta con serenidad las dificultades } \\
\text { institucionales }\end{array}$ & 3 & 100 & Ser & - & - & 100 \\
\hline $\begin{array}{l}\text { Diseña políticas para promover } \\
\text { la integración }\end{array}$ & 3 & 100 & Hacer & - & - & 100 \\
\hline $\begin{array}{l}\text { Fomenta una buena comunicación } \\
\text { a lo interno }\end{array}$ & 1 & 33.33 & Ser & 2 & 66.66 & 100 \\
\hline Facilita la comunicación con el entorno & 1 & 33.33 & Hacer & 2 & 66.66 & 100 \\
\hline Asume críticas a su gestión & 2 & 66.66 & Ser & 1 & 33.33 & 100 \\
\hline $\begin{array}{l}\text { Participa en la orientación hacia } \\
\text { la tarea y las relaciones }\end{array}$ & - & - & Convivir & - & - & - \\
\hline Interpreta la delegación de Autoridad & 2 & 66.66 & Conocer & 1 & 33.33 & 100 \\
\hline Todas las anteriores & 1 & 33.33 & - & 2 & 66.66 & 100 \\
\hline Ninguna de las anteriores & - & - & - & - & - & - \\
\hline
\end{tabular}

Fuente: Elaboración propia basada en los datos obtenidos del instrumento ECAD.

sonal para incorporarlos a la evaluación, esto se identifica con el saber conocer y las competencias técnicas. Los resultados constituyen una fortaleza en función de los enunciados presentados para este saber, dos (2) de los directores realizan esta actividad.

Con relación al saber hacer, el $33.33 \%$ utiliza asistentes para establecer controles institucionales, el $66.66 \%$ la interacción en los procesos consciente de su aporte a la labor supervisora, elabora parámetros de medición asociados a la productividad académica de los docentes, facilita el control en áreas bajo su responsabilidad, detalla controles presupuestarios y evalúa resultados, mientras el 100 afirmó que aplica acciones correctivas cuando lo cree prudente. Los resultados apuntan a la aplicación de mecanismos de control en la organización; se evidencian en este caso las competencias metodológicas.

En relación con el proceso de control, el mismo constituye una garantía para lograr en términos reales los resultados en función de las actividades planificadas, por ello la aplicación de medidas correctivas determina el avance hacia el logro de metas y objetivos. La dinámica organizacional y la complejidad ofrecidas por el entorno ratifica la importancia de esta función, sobre todo si se asume la premisa administrativa planteada por 
Acción directiva: Un enfoque centrado en competencias

Zambrano Flores, Blanca M. y Marval Galvis, Elina V.

Hernández (2005:344) quien señala "sólo lo que se mide se puede mejorar". Claro está, se sugiere asumir la medición no en términos del "dato", sino en lo que subyace por debajo del mismo.

En cuanto al saber ser, el $100 \%$ expresó que humanizan los mecanismos de control en los procesos bajo su responsabilidad. Los resultados asociados con este saber, demuestran las competencias sociales en la acción directiva por ellos desarrolladas, pues al momento de aplicar mecanismos de control toman en cuenta al individuo como ser humano, sus aciertos y errores al realizar sus tareas. Esto se relaciona con el saber convivir, por cuanto el total de los directores afirman participar en forma conjunta con el empleado en su evaluación, es decir, se involucran en dicho proceso. Se infiere aunque de forma incipiente acciones para desarrollar competencias participativas, tal como se observa (Tabla 4).

\subsection{Competencias: una propuesta alternativa}

Por naturaleza el ser humano es social, ello induce a vivir de forma organizada. Posiblemente, esto es parte de la cultura de lo individual y lo fragmentario transferida a las diversas áreas del conocimiento, sin profundizar en las razones muchas de carácter didáctico o metodológico. Para las investigadoras, ello justifica la especialización y establece distancia con la visión de integralidad o de conjunto. En esta perspectiva, la administración es influenciada por el mecanicismo, su instrumental adquiere carácter técnico y quien lo utiliza es un especialista que aplica las técnicas para hacer eficiente el trabajo; pertinente entonces es preguntarse ¿y el carácter humano? Se administra por el hombre y para el hombre ¿cómo separar esta visión de su acción?

La propuesta intenta responder a los cambios para alcanzar una experiencia centrada en competencias en el ámbito administrativo universitario, donde se manifieste el conocimiento de sí mismo, de los demás y el compromiso de un trabajo honesto, identificado con lo social. La misma se justifica por:

1. La necesidad de cambios presentes en el sistema educativo.

2. La responsabilidad de orientar los niveles educativos precedentes.

3. La responsabilidad social de la universidad para contribuir con el desarrollo sostenible y sustentable.

4. Viabilizar en la práctica la asunción de nuevos paradigmas de la ciencia y en este particular de los enfoques directivos.

5. En el caso de la UNERMB, posibilitar en lo concreto los elementos anteriores, al promover una acción directiva que impulse los cambios (Diagrama 4).

\subsection{Lineamientos que orientan la propuesta}

La propuesta centrada en competencias constituye un intento por armonizar competencias, saberes y funciones administrativas, en el propósito de lograr planificar, organizar, dirigir y controlar, con una visión humanista que viabilice transformar la realidad administrativa, pero simultáneamente debe producir cambios en la condición humana observables en su desarrollo intelectual y emocional con el propósito de generar creati- 
Tabla 4

Competencias a desarrollar en el proceso de control

\begin{tabular}{lcccccc}
\hline & \multicolumn{7}{c}{ Considera } & \multicolumn{3}{c}{ No considera } & \\
\hline \multicolumn{1}{c}{ Competencias } & F & $\%$ & Saber & F & $\%$ & Total (\%) \\
\hline Humaniza el control en los Procesos & 3 & 100 & Ser & - & - & 100 \\
Utiliza la interacción en los procesos & 2 & 66.66 & Hacer & 1 & 33.33 & 100 \\
Elabora parámetros de Medición & 2 & 66.66 & Hacer & 1 & 33.33 & 100 \\
Estudia aspectos del desempeño & & & & & & \\
del personal & 2 & 66.66 & Conocer & 1 & 33.33 & 100 \\
Aplica acciones correctivas & 3 & 100 & Hacer & - & - & 100 \\
Participa con el empleado en su & & & & & & \\
evaluación & 3 & 100 & Convivir & - & - & 100 \\
Facilita el control & 2 & 66.66 & Hacer & 1 & 33.33 & 100 \\
Utiliza asistente para establecer & & & & & & \\
control & 1 & 33.33 & Hacer & 2 & 66.66 & 100 \\
Detalla controles presupuestarios & 2 & 66.66 & Hacer & 1 & 33.33 & 100 \\
Evalúa resultados & 2 & 66.66 & Hacer & 1 & 33.33 & 100 \\
Todas las anteriores & - & - & - & - & - & - \\
Ninguna de las anteriores & - & - & - & - & - & - \\
\hline
\end{tabular}

Fuente: Elaboración propia basada en los datos obtenidos del instrumento ECAD.

\section{Diagrama 4}

Iniciativa y justificación

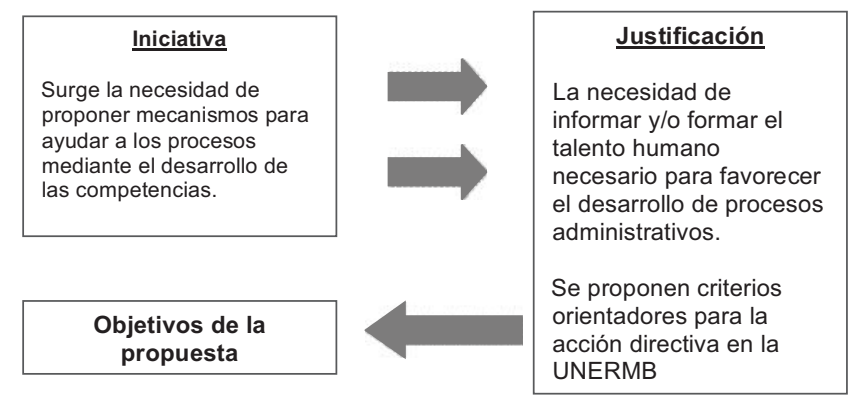

Fuente: Elaboración propia.

vidad, confianza y comprensión. Se deben tomar en cuenta como plantea Zambrano (2006b:15) "los tres (3) elementos que al ser parte de sus competencias inciden significativamente sobre su motiva- ción: saber, poder y querer", todos asociados a la condición humana de quien ejecuta la acción directiva.

Se plantean acciones direccionadas por el saber conocer, saber hacer, 
Acción directiva: Un enfoque centrado en competencias

Zambrano Flores, Blanca M. y Marval Galvis, Elina V.

saber ser y saber convivir, entre estos la conformación de talleres y conversatorios como estrategias para viabilizar la reflexión a partir del interactuar, potencian conjugar la teoría y la práctica en un espacio apto para reflexionar, pensar y hacer. En el propósito de lograr su intencionalidad, se recomienda que éstos sean:

1. Direccionados por especialistas, sin desconocer los saberes populares, orientados al crecimiento personal y profesional.

2. Monitoreados de forma permanente para verificar cambios o adopción de actitudes alineadas con la orientación contenida en la propuesta.

3. Centrados en competencias, con enfoque humanista como modalidad exitosa para promover los cambio deseados. Se sugiere la realización de talleres y conversatorios destinados a proporcionar información y/o formación de los Directores de Programas Académicos de la UNERMB.

Se prevé con la propuesta alcances en el término de las competencias: técnicas, metodológicas, sociales y participativas, pues se tiene como norte, no sólo incidir en lo administrativo, sino en la condición humana de quienes direccionan, guiados por principios y valores como: cooperación, respeto, solidaridad, responsabilidad, honestidad, equidad y en la aspiración actual de la administración pública, la corresponsabilidad, la rendición de cuentas y la transparencia. En este sentido, se plantean los siguientes lineamientos para su asunción en la propuesta:

1. Acción directiva en el marco del humanismo.

2. La administración como un enfoque alternativo al servicio del hombre.
3. La educación como servicio público.

4. Integración de los componentes institucionales.

5. Sinergia como elemento articulador intra interinstitucional.

6. Cooperación como principio en búsqueda de la excelencia.

7. Innovación y creatividad en los procesos administrativos.

8. Promoción para la participación de los distintos actores.

9. Articulación de los saberes en el desempeño directivo.

10. Actuación dentro de los principios constitucionales y el marco normativo institucional.

11. Vinculación permanente con sectores educativos, empresariales y comunidades.

12. Priorizar lo colectivo por encima de lo individual.

Los lineamientos a los cuales se hace referencia se articulan a los fundamentos teóricos que orientan el estudio, asociados a la búsqueda del crecimiento humano y profesional de quien ejerce tal actividad en la universidad.

\section{Conclusiones}

Es importante resaltar que quienes ejecutan la acción directiva en los Programas Académicos donde se imparte formación profesional a nivel de pregrado en la UNERMB, reconocen su autoformación en el área administrativa, lo cual supone deficiencias cognitivas que inciden en su actuación.

Examinar la acción directiva, permitió reconocer las características de sus actores y la detección de una realidad particularmente contradictoria, pues és- 
tos no asumen de forma manifiesta la dirección, componente humano del proceso administrativo, por el contrario, enfatizan las funciones mecanicistas, planificación, organización y control, por lo que se debate de forma permanente en una realidad dialógica: asumir el cambio o mantenerse en la cotidianidad administrativa presente en la UNERMB.

Los resultados obtenidos con respecto a las habilidades humanas que exhibe en su acción el director (comunicación, motivación, toma de decisiones y liderazgo) ratifican la realidad dialógica en la cual se debate su acción.

La acción directiva en la UNERMB se produce en la perspectiva del enfoque tradicional, sin una clara comprensión de las competencias de las cuales dispone, ni tampoco su relación con los saberes de los cuales dispone quienes las ejecutan.

En las funciones: planificación, organización, dirección y control, se observan limitación en los saberes, poca armonía y dominio en las competencias técnicas, metodológicas, sociales y participativas. Para la dirección, se da la prevalencia de competencias sociales y participativas, mayormente identificadas con la tendencia humanista de la administración, por lo cual se requiere una acción directiva con este carácter que incluya una visión alternativa de la administración desarrollada con apropiación del contexto nacional, regional, local e institucional para favorecer el uso pleno de las competencias.

\section{Referencias Bibliográficas}

Ballina, Fernando (2000). Teoría de la administración. Un enfoque alternativo. México: McGraw Hill.

Benavides, Olga (2002). Competencias y competitividad. Diseño para organizaciones latinoamericanas. Colombia: Editorial Normas. S. A.

Chiavenato, Idalberto (2002). Gestión del talento humano. El nuevo papel de recursos humanos en las organizaciones. Colombia: McGraw Hill.

Delors, Jacques (1996). Informe Delors. La educación encierra un tesoro. Madrid: UNESCO. Santillana.

Guédez, Víctor (1998). Gerencia, cultura y educación. Caracas: Fondo editorial Tropykos.

Hernández, Sergio (2005). Administración, pensamiento, proceso, estrategia y vanguardia. México: Mc Graw Hill.

Maldonado, Miguel (2006). Las competencias, una opción de vida. Metodología para el diseño curricular. Colombia: Digiprint, editores.

Núñez, Alberto (2000). La innovación tecnológica en la industria petrolera. $\mathrm{Ca}$ racas: Ministerio de Ciencia y Tecnología.

Pérez, Miguel (2005). Desarrollo de las habilidades del personal directivo en instituciones educativas. México: editorial Trillas.

Tobón, Sergio (2006). Formación basada en competencias. Pensamiento complejo, diseño curricular y didáctica. Colombia: Ecoe, ediciones LTDA.

Villarroel, César (2005). "Gerencia, planificación y evaluación universitaria”. Revista Educere. Año, No. 13. Mérida: Gráficas El Porta título. 
Acción directiva: Un enfoque centrado en competencias

Zambrano Flores, Blanca M. y Marval Galvis, Elina V.

Zambrano, Blanca (2006a). Cambio y ejercicio docente: un binomio necesario en la educación superior. Informe No. 1. Maracaibo: Tecana American University. Doctorate of Education in Educational Administration.

Zambrano, Blanca (2006b). El directivo y sus habilidades en el contexto educativo. Informe No. 3. Maracaibo: Tecana American University. Doctorate of Education in Educational Administration.
Zambrano, Blanca (2003). La acción gerencial en el contexto de la mediana empresa industrial. Municipio Lagunillas: perspectiva actual. Trabajo de ascenso no publicado. Cabimas. Universidad Nacional Experimental "Rafael María Baralt". 